\title{
Mueller matrix microscope with a dual continuous rotating compensator setup and digital demodulation
}

\author{
Oriol Arteaga, ${ }^{*}$ Marta Baldrís, Joan Antó, Adolf Canillas, \\ Esther Pascual, and Enric Bertran \\ Feman Group, Dep. Física Aplicada i Òptica, IN2UB, C/Martí i Franquès 1, Universitat de Barcelona, Catalonia, Spain \\ ${ }^{\star}$ Corresponding author: oarteaga @ub.edu
}

Received 18 December 2013; revised 19 February 2014; accepted 23 February 2014; posted 24 February 2014 (Doc. ID 203112); published 31 March 2014

\begin{abstract}
In this paper we describe a new Mueller matrix (MM) microscope that generalizes and makes quantitative the polarized light microscopy technique. In this instrument all the elements of the MU are simultaneously determined from the analysis in the frequency domain of the time-dependent intensity of the light beam at every pixel of the camera. The variations in intensity are created by the two compensators continuously rotating at different angular frequencies. A typical measurement is completed in a little over one minute and it can be applied to any visible wavelength. Some examples are presented to demonstrate the capabilities of the instrument. (C) 2014 Optical Society of America

OCIS codes: (120.2130) Ellipsometry and polarimetry; (120.5410) Polarimetry; (240.2130) Ellipsometry and polarimetry; (120.5060) Phase modulation.

http://dx.doi.org/10.1364/AO.53.002236
\end{abstract}

\section{Introduction}

Polarized light microscopy is widely used to observe specimens that have an optically anisotropic character. It has a vast number of applications [1-3], for example for studying rocks and minerals in geology, natural and synthetic polymer chains in chemistry, organic tissues in biology, etc. In most of these applications the main optical effect under study is the linear birefringence. A drawback of this instrument is that it essentially provides qualitative information since it does not readily offer quantitative measurements of the spatial distribution of the optical anisotropy of the specimen $[\underline{4}, \underline{5}]$. Approaches based on the comparison of the interference polarization colors with the Michel-Levy interference color chart, or on getting the maximum darkness when using a Berek compensator, are only semi-quantitative since, in most configurations, they ultimately rely on the

$1559-128 \mathrm{X} / 14 / 102236-10 \$ 15.00 / 0$

(C) 2014 Optical Society of America visual and technical skills of the microscope user. Mueller matrix (MM) microscopy is the natural generalization of polarized light microscopy because it brings quantitative results and allows the simultaneous determination of linear birefringence, linear dichroism, circular birefringence, circular dichroism, and depolarization [6-11].

The experimental approaches to measure a complete MM can be classified according to the type of instrumental setup used for the measurement: using dual rotating compensators $[\underline{12}, \underline{13}]$, four photoelastic modulators [14], two or more liquid crystal variable retarders [15], or beam splitters along with divisionof-amplitude method [16,17]. In principle, any of these four approaches can be applied to obtain complete MM measurements in different experimental arrangements: ellipsometers, scatterometers, transmission, and reflection microscopes. If we restrict our attention to imaging devices, i.e., instruments in which cameras with CCD or CMOS sensors are used as detectors, only three of these four techniques are directly applicable because cameras cannot follow 
the high modulation speed of photoelastic modulators (in the $\mathrm{kHz}$ range). Currently, some efforts are being carried out to allow the use of photoelastic modulators in imaging systems to take advantage of their optimal characteristics as sources of polarization modulation. But this requires encoding a complicate demodulation pattern in the strobed light emitted by an LED source, a technique that is not easy to implement $[\underline{18}, \underline{19}]$.

In this paper we describe an MM microscope that uses two continuously rotating compensators. The dual rotating compensator technique has been known since the late 70s [20-22] and currently it is offered in many commercial $\mathrm{M} \overline{\mathrm{M}}$ ellipsometers. However, the novelty of the MM microscope we propose lies in the use of the signal coming from a camera to implement the analysis in frequency of the modulation introduced by the two continuously rotating compensators. The intensity detected by every pixel of the camera is frequency analyzed by digital demodulation. To our knowledge, this is the first time it has been done, as the typical strategy used by imaging instruments that measure complete MMs was based in the combination of the intensities measured at several fixed orientations of the compensators [23] or the liquid crystals [24,25]. This sequential, "step-by-step" method is well established and it is relatively easy to implement because the camera only needs to take an image at some predetermined positions of the compensators (usually 16 different positions). The whole process is fast and, computationally wise and not very demanding because only a few frames are captured. However, the technique we use, consisting of digital demodulation of thousands of continuously captured frames, offers higher accuracy and sensitivity because it incorporates a complete analysis in the frequency domain.

Here we report the design, the working principles, the calibration, and some measurement examples of an MM microscope with a dual continuous rotating compensator setup and digital demodulation.

\section{Working Principles}

The most convenient way to describe the polarization effects of optical elements is the Stokes-Mueller calculus. We will use this formalism to describe the state of polarization of the light beam as it traverses all the polarization-changing optical elements.

In the MM microscope, the polarization state generator (PSG) and the polarization state analyzer (PSA) are composed of a polarizer and a rotating compensator. The MM of a rotating compensator, $\mathbf{M}_{C}$, is

$$
\mathbf{M}_{C}(\theta, \delta)=\left[\begin{array}{cccc}
1 & 0 & 0 & 0 \\
0 & C_{2 \theta}^{2}+S_{2 \theta}^{2} C_{\delta} & C_{2 \theta} S_{2 \theta}\left(1-C_{\delta}\right) & -S_{2 \theta} S_{\delta} \\
0 & C_{2 \theta} S_{2 \theta}\left(1-C_{\delta}\right) & S_{2 \theta}^{2}+C_{2 \theta}^{2} C_{\delta} & C_{2 \theta} S_{\delta} \\
0 & S_{2 \theta} S_{\delta} & -C_{2 \theta} S_{\delta} & S_{\delta}
\end{array}\right],
$$

where we have employed the short notation

$$
\begin{aligned}
& S_{X} \equiv \sin (X), \\
& C_{X} \equiv \cos (X) .
\end{aligned}
$$

$\delta$ is the retardation introduced by compensator and $\theta$ is the orientation of its fast axis. Even for compensators sold as "achromatic," $\delta$ still changes slightly with the wavelength. $\theta$ evolves during the measurement due to the rotating setup. Its time dependence is given by

$$
\theta(t)=\omega t+\phi,
$$

where $\omega$ is the angular speed, which is a constant during the measurement, and $\phi$ is the phase, i.e., the initial orientation of the compensator.

The light entering the PSG, $\mathbf{S}_{\text {input }}$, passes first through a polarizer, $\mathbf{P}_{0}$, and then goes through the first rotating compensator, $\mathbf{M}_{C 0}(t)$. After being transmitted through the sample, $M_{S}$, it is collected by the objective and sent to the PSA, in which it goes through the second rotating compensator, $\mathbf{M}_{C 1}(t)$, and a analyzer, $\mathbf{P}_{1}$. The Stokes vector at the detector, $\mathbf{S}_{\text {out }}$, is given by the following matrix multiplication:

$$
\mathbf{S}_{\text {out }}(t)=\mathbf{P}_{1} \mathbf{M}_{C 1}(t) \mathbf{M}_{S} \mathbf{M}_{C 0}(t) \mathbf{P}_{0} \mathbf{S}_{\text {input }},
$$

where without lost of generality $\mathbf{S}_{\text {input }}$ can be considered as unpolarized light. In our setup the polarizers are oriented at $90^{\circ}$ to each other. We chose the crossed polarizers configuration because it allows a very precise orientation of the polarizers during the instrument construction, but the MM microscope can work with arbitrary orientations of the polarizer and analyzer, as long as they are well known. Each compensator has its own time dependence because they rotate at different speeds. The MM of the sample is given by

$$
\mathbf{M}_{S}=\left[\begin{array}{cccc}
m_{00} & m_{01} & m_{02} & m_{03} \\
m_{10} & m_{11} & m_{12} & m_{13} \\
m_{20} & m_{21} & m_{22} & m_{23} \\
m_{30} & m_{31} & m_{32} & m_{33}
\end{array}\right]
$$

In general, every MM element of the sample is not related to a single optical property and there are analytical methods to accurately extract all the properties. However, for a first qualitative interpretation (mostly valid in the limit of weak optical anisotropies), it is useful to assume that $m_{01}, m_{02}$, $m_{10}$, and $m_{20}$ are due to linear dichroism, $m_{03}$ and $m_{30}$ refer to circular dichroism, $m_{12}$ and $m_{12}$ are linked circular birefringence, and $m_{13}, m_{23}, m_{31}$, $m_{32}$ are associated to linear birefringence. In the calibration configuration, where no sample is examined, none of these effects is present and $\mathbf{M}_{S}$ coincides with the identity matrix. 
The intensity measured at every pixel of the camera is given by the first element of the Stokes vector in Eq. (4), which can be written as

$I(t)=\left[\begin{array}{c}1 \\ C_{2 \theta_{0}}^{2}+C_{\delta_{0}} S_{2 \theta_{0}}^{2} \\ C_{2 \theta_{0}} S_{2 \theta_{0}}\left(1-C_{\delta_{0}}\right) \\ S_{\delta_{0}} S_{2 \theta_{0}} \\ -\left(C_{2 \theta_{1}}^{2}+C_{\delta_{1}} S_{2 \theta_{1}}^{2}\right) \\ -\left(C_{2 \theta_{0}}^{2}+C_{\delta_{0}} S_{2 \theta_{0}}^{2}\right)\left(C_{2 \theta_{1}}^{2}+C_{\delta_{1}} S_{2 \theta_{1}}^{2}\right) \\ -\left(C_{2 \theta_{0}} S_{2 \theta_{0}}\left(1-C_{\delta_{0}}\right)\right)\left(C_{2 \theta_{1}}^{2}+C_{\delta_{1}} S_{2 \theta_{1}}^{2}\right) \\ -\left(S_{\delta_{0}} S_{2 \theta_{0}}\right)\left(C_{2 \theta_{1}}^{2}+C_{\delta_{1}} S_{2 \theta_{1}}^{2}\right) \\ -C_{2 \theta_{1}} S_{2 \theta_{1}}\left(1-C_{\delta 1}\right) \\ -\left(C_{2 \theta_{0}}^{2}+C_{\delta_{0}} S_{2 \theta_{0}}^{2}\right)\left[C_{2 \theta_{1}} S_{2 \theta_{1}}\left(1-C_{\delta 1}\right)\right] \\ -\left[C_{2 \theta_{0}} S_{2 \theta_{0}}\left(1-C_{\delta 0}\right)\right]\left[C_{2 \theta_{1}} S_{2 \theta_{1}}\left(1-C_{\delta 1}\right)\right] \\ -S_{\delta_{0}} S_{2 \theta_{0}}\left[C_{2 \theta_{1}} S_{2 \theta_{1}}\left(1-C_{\delta 1}\right)\right] \\ S_{\delta_{1}} S_{2 \theta_{1}} \\ S_{\delta_{1}} S_{2 \theta_{1}}\left[C_{2 \theta_{0}}^{2}+C_{\delta_{0}} S_{2 \theta_{0}}^{2}\right] \\ S_{\delta_{1}} S_{2 \theta_{1}}\left[C_{2 \theta_{0}} S_{2 \theta_{0}}\left(1-C_{\delta 0}\right)\right] \\ S_{\delta_{1}} S_{2 \theta_{1}} S_{\delta_{0}} S_{2 \theta_{0}}\end{array}\right]^{T}\left[\begin{array}{l}m_{00} \\ m_{01} \\ m_{02} \\ m_{03} \\ m_{10} \\ m_{11} \\ m_{12} \\ m_{13} \\ m_{20} \\ m_{21} \\ m_{22} \\ m_{23} \\ m_{30} \\ m_{31} \\ m_{32} \\ m_{33}\end{array}\right]$

Every compensator has its own time dependence: $\theta_{0}(t)=\omega_{0} t+\phi_{0}$ and $\theta_{1}(t)=\omega_{1} t+\phi_{1}$. The election of favorable angular speeds for the rotating compensators is discussed in the next section.

The most common approach to solve the dual rotating compensator problem, i.e., to obtain the MM elements of the sample from the measured timevarying intensity, consists of a straightforward Fourier analysis of the detected signal $[12,22,26,27]$. Briefly, this method is only applicable if $\omega_{0}$ and $\omega_{1}$ are exact multiples of a certain frequency $\omega$; for example $\omega_{0}=p \omega$ and $\omega_{1}=q \omega$, being $p$ and $q$ integers. When this happens all the multiplications of trigonometric functions shown in Eq. (6) can be expanded as a Fourier series centered in $\omega$ and containing many harmonic terms. Every Fourier coefficient is then related to linear combination of MM elements and the whole ensemble forms a complicate linear system with 15 independent equations. Once the system is inverted, the values of the MM elements can be recovered.

Here we follow a different approach to apply the digital demodulation. This alternative method was already used by Ichimoto et al. [13] for a dual rotating compensator instrument with a single point detector. A similar procedure is also applied in instruments based on multiple photoelastic modulators [14]. It permits us to disclose the values the MM elements more straightforwardly, and it does not require the ratio $\omega_{0} / \omega_{1}$ to be a rational number. We start the description of this procedure noting that, in vector notation, Eq. (6) can be rewritten as

$$
I(t)=\mathbf{B}^{T}(t) \mathbf{A},
$$

where $\mathbf{A}$ is a 16 -element vector containing all the MM elements of the sample and $\mathbf{B}(t)$ is what we call the basis vector that evolves with time. If a new dimension is added, this equation can be generalized to include in the same calculation all the intensities recorded over time:

$$
\mathbf{I}=\mathbf{B}^{T} \mathbf{A},
$$

where the time dependence is no longer specified. I is a vector of $N$ elements, $N$ being the number of raw images captured by the camera overtime. Here B is no longer a vector but a matrix with dimension $N \times 16$. Its elements are given by

$$
\begin{aligned}
B_{j, 0}= & 1, \\
B_{j, 1}= & \cos ^{2}\left(2\left(\omega_{0} j \Delta t+\phi_{0}\right)\right) \\
& \left.+\cos \left(\delta_{0}\right) \sin ^{2}\left(2\left(\omega_{0} j \Delta t+\phi_{0}\right)\right)\right), \\
B_{j, 2}= & \cos \left(2\left(\omega_{0} j \Delta t+\phi_{0}\right)\right) \\
& \times \sin \left(2\left(\omega_{0} j \Delta t+\phi_{0}\right)\right)\left(1-\cos \delta_{0}\right), \\
B_{j, 3}= & \sin \left(\delta_{0}(j \Delta t)\right) \cos \left(\delta_{0}(j \Delta t)\right), \\
{[\ldots] } & \\
B_{j, 15}= & \sin \left(\delta_{0}(j \Delta t)\right) \cos \left(\delta_{1}(j \Delta t)\right) \\
& \times \cos \left(\delta_{2}(j \Delta t)\right) \sin \left(\delta_{3}(j \Delta t)\right),
\end{aligned}
$$

where $j=0, \ldots, N-1 . \Delta t$ is the inverse of the frame rate of the camera and $N \Delta t$ is the total time of data capturing.

If we multiply both sides of Eq. (ㅁ) by $\mathbf{B}$ we get

$$
\mathbf{B I}=\mathbf{B B}^{T} \mathbf{A},
$$

and from here we can deduce

$$
\mathbf{A}=\left(\mathbf{B B}^{T}\right)^{-1} \mathbf{B I}=\mathbf{K B I},
$$

where $\mathbf{K} \equiv\left(\mathbf{B B}^{T}\right)^{-1}$ is a matrix of dimension $16 \times 16$. Equation (11) shows the matrix calculation needed to obtain the MM elements as a function of the measured intensities contained in I. This equation needs to be applied for the time-varying intensities recorded at every pixel of the camera. However the matrices $\mathbf{B}$ and $\mathbf{K}$ are valid for all the pixels and, therefore, the calculation of these matrices, which is the computationally most time-consuming task, needs to be done only once. The optical properties of the polarizer and the compensators must be homogeneous all over the surface of the sample to assure that $\mathbf{B}$ and $\mathbf{K}$ can be taken as constants for all the images. The resolution of the camera and the number of images captured, $N$, will determine the computing time needed to extract the MM parameters from measurements. In our MM microscope, the calculation time is typically much shorter than the acquisition time, so that the final MM image is obtained right after the measurement finishes. Moreover, in modern computers with multi-core processors, this calculation is greatly optimized if it is programmed to run in parallel using all cores. More details about 


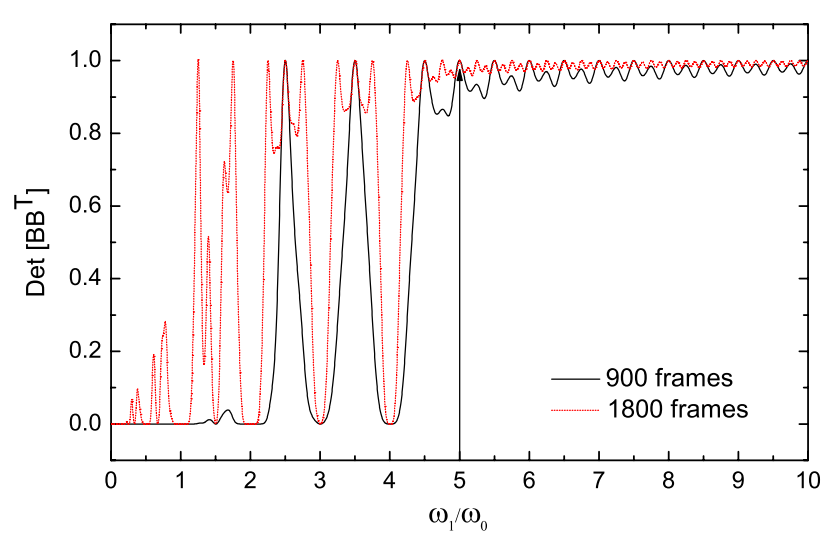

Fig. 1. Values of the determinant $\left|\mathbf{B B}^{T}\right|$ normalized to its maximum value as a function of the ratio of the angular speeds of the two compensators, $\omega_{1} / \omega_{0}$. All the ratio values where the determinant drops to zero should be avoided because there $\mathbf{B B}^{T}$ becomes a singular matrix. The arrow indicates the $\omega_{1} / \omega_{0}=5$ that we use in measurements.

the processing time of our instrument are given in the next section.

The rotation speed of the first and second compensators are the two basic parameters that control how the detected intensity varies over time. The analysis of the optimum rotation speeds for a dual rotating compensator setup has been considered in previous publications $[13,27]$. The main conclusion is that there are some specific values of the ratio $\omega_{1} / \omega_{0}$ that should be avoided. This happens because for some values of this ratio the determinant of the matrix $\mathbf{B B}^{T}$ goes to zero and therefore it is not invertible. The inverted matrix $\mathbf{K}$ is best conditioned when the determinant of $\mathbf{B B}^{T}$ takes its maximum values. In Fig. 1 we have represented the variation of $\left|\mathbf{B B}^{T}\right|$ as a function $\omega_{1} / \omega_{0}$ for two datasets of 900 and 1800 frames with $\Delta t=0.04 \mathrm{~s}$. Clearly a speed ratio of, for example, 2 or 3 would be unsuitable for measurement, on the other hand, there are many ratio choices $(2.5,3.5,5$, etc) that would work equally well.

\section{Instrument Description}

The whole instrument is mounted vertically, with light traveling from bottom to top following the usual design of transmission microscopes. A scheme of the optical design and a photo of the instrument are displayed in Fig. 2. The light source is a white LED illuminator (Metaphase MP-LED-150) that emits light in all the visible range. The light beam is collimated by a lens and then it goes through a bandpass interference filter that selects the wavelength of the measurement. A $45^{\circ}$ mirror redirects the beam in the vertical direction toward the PSG.

The PSG and the PSA are composed of two elements: an achromatic compensator (quarter-wave polymer retarder film, Edmund optics) and a polarizer (high-contrast linear polarizing film, Edmund optics). We use film elements because they offer a large aperture, and we have tested experimentally that their retardation homogeneity is better than

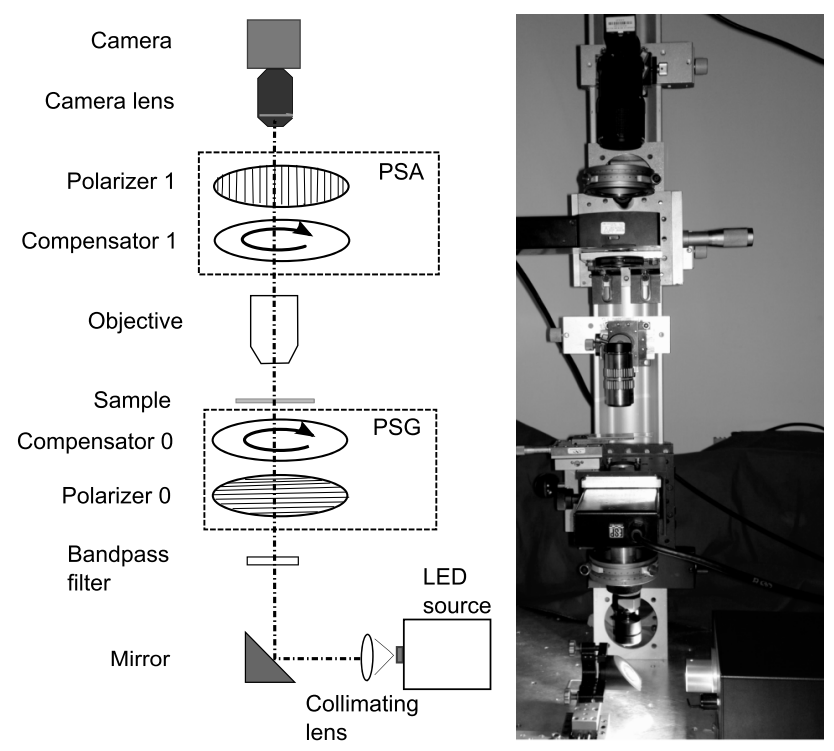

Fig. 2. Scheme and photo of the optical setup of the MM microscope.

in retarders made of crystals, leading to a better MM imaging. Only in the case of measurements in the UV (below $400 \mathrm{~nm}$ ) would it be advisable to replace film polymer elements by crystal polarization ones, because the performance of the film polymer elements quickly become far from ideal in the UV. The instrument can be operated with compensators of almost any retardation, as long as it is not too small [13]. Each compensator is mounted in a precision, motorized rotation stage (Newport URS75BCC), which offers full control of the motor position and of the rotation speed (up to $80^{\circ} / \mathrm{s}$ ). The polarizers are mounted in a manual rotation stages (Newport UTR80S). The sample and the microscope objective are placed in between the PSG and PSA. The objective is an infinite-corrected long working distance objective (Edmund Optics), which is mostly free from strain-induced linear birefringence. The whole setup is on the top of an anti-vibration table (MinusK).

The detection system is composed of a camera (Ueye UI-122x) and a telecentric camera objective. This camera has a resolution of $752 \times 480(0.36$ MP). Keeping a constant frame rate of 50 frames per second (fps) it allows us to vary the exposure time from 0.1 to $19.9 \mathrm{~ms}$. If a samples requires a longer exposure it can still be examined by decreasing the frame rate and reducing the rotation speed of the compensators. The camera and the motor controllers (Newport SMC100) are connected to a personal computer (with a Intel Core 2 Quad Q6600 processor) that operates the instrument using Labview.

In our most usual configuration, the camera acquires images at approximately $50 \mathrm{fps}$, the first compensator rotates at $\omega_{0}=5^{\circ} / \mathrm{s}(0.0138 \mathrm{~Hz})$, and the second at $\omega_{1}=25^{\circ} / \mathrm{s}(0.0694 \mathrm{~Hz})$. Thus, we have a ratio $\omega_{1} / \omega_{0}=5$ where, as shown in Fig. 1 , the determinant $\left|\mathbf{B B}^{T}\right|$ takes maximum values. The number of collected frames is adjusted so that, at least, 
a complete turn of the slowest compensator is recorded (and therefore five complete turns of the fastest one). In this case this means acquiring 3600 frames of data, which takes around $72 \mathrm{~s}$. As the frame rate of the camera can suffer small oscillations, it is convenient to programmatically measure the $\Delta t$ corresponding to each frame in real-time and use it for the calculation in Eq. (9).

The data acquisition is programmed so that the motors always start at a certain precise orientation of the compensators, which we have chosen arbitrarily. Therefore, the angles $\phi_{0}$ and $\phi_{1}$, describing the misalignment of the fast axes of the compensators with respect to these two orientations, are constants determined upon calibration. To assure that $\phi_{0}$ and $\phi_{1}$ can really be treated as constants, it is important to trigger the camera capture with the data acquisition. Any random delay in the initialization of the camera would result in random variations of $\phi_{0}$ and $\phi_{1}$, that would ultimately translate in a poor determination of some MM elements. The angular precision in the determination of $\phi_{0}$ and $\phi_{1}$ is typically limited by the factor $\Delta t \omega_{i}$. This means that a compromise between the camera frame rate and the rotation speed of the compensators needs to be found to determine accurately $\phi_{0}$ and $\phi_{1}$. In general, it is advisable to avoid very high rotation speeds, not only because they would require an ultrafast camera, but also because they tend to induce undesired vibrations in the setup. Given our typical frame rate of $50 \mathrm{fps}$
$(\Delta t=0.02 \mathrm{~s})$, we have a precision around $0.1^{\circ}$ for $\phi_{0}$ and around $0.5^{\circ}$ for $\phi_{1}$. In the next section we discuss how these angles are measured and how the errors in their determination propagate over all the MM elements.

\section{Calibration}

The objective of the calibration is the determination of the offset phase angles $\phi_{0}$ and $\phi_{1}$ and the retardation values $\delta_{0}$ and $\delta_{1}$ of the compensators. $\delta_{0}$ and $\delta_{1}$ vary with the wavelength because the compensators are not fully achromatic. The calibration is done without sample and removing the microscope objective from the setup to ensure that there are no strains that perturb the signal detected. During the calibration the camera is used as a single point detector by averaging the intensity given by all the pixels.

When there is no sample, the time-depending intensity is given by

$$
\begin{aligned}
I(t)= & 1-\left(C_{2 \theta_{0}}^{2}+C_{\delta_{0}} S_{2 \theta_{0}}^{2}\right)\left(C_{2 \theta_{1}}^{2}+C_{\delta_{1}} S_{2 \theta_{1}}^{2}\right) \\
& -\left[C_{2 \theta_{0}} S_{2 \theta_{0}}\left(1-C_{\delta 0}\right)\right]\left[C_{2 \theta_{1}} S_{2 \theta_{1}}\left(1-C_{\delta 1}\right)\right] \\
& +S_{\delta_{1}} S_{2 \theta_{1}} S_{\delta_{0}} S_{2 \theta_{0}} .
\end{aligned}
$$

If $\phi_{0}, \phi_{1}, \delta_{0}$, and $\delta_{1}$ are perfectly known, this timedepending intensity will be analyzed as the identity matrix. Now we can examine what happens if these

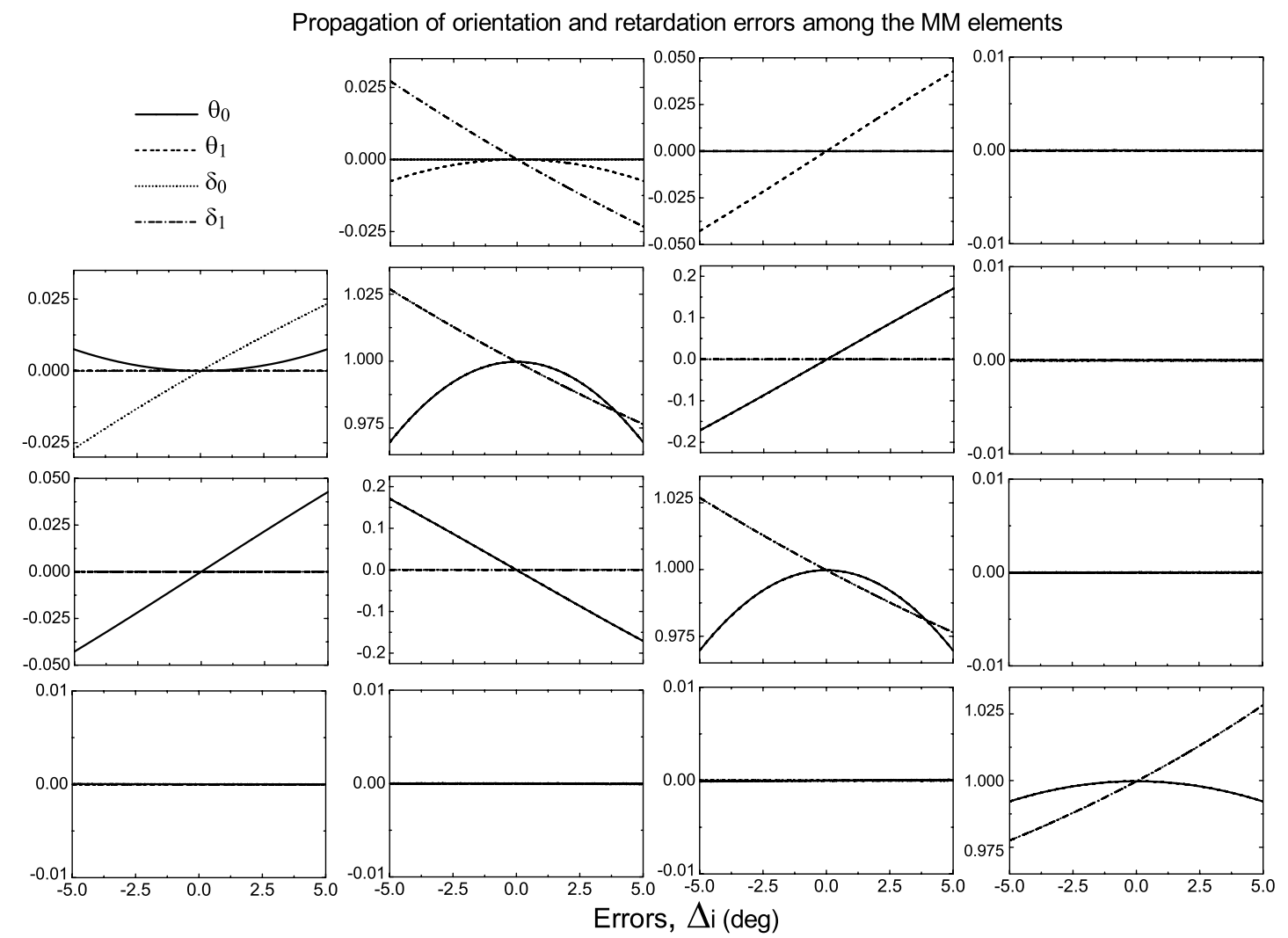

Fig. 3. Simulation of how errors of $\theta_{0}, \theta_{1}, \delta_{0}$, and $\delta_{1}$ in the interval between $\pm 5^{\circ}$ propagate among the elements of an MM. 
parameters have a certain error, i.e., if we do not know with precision the orientation and retardation values. This can be simulated by assuming that each term has an associated error $\Delta_{i}: \theta_{0}=\theta_{0}^{\prime}+\Delta_{\phi 0}$, $\theta_{1}=\theta_{1}^{\prime}+\Delta_{\phi 1}, \delta_{0}=\delta_{0}+\Delta_{\delta 0}$, and $\delta_{1}=\delta_{1}+\Delta_{\delta 1}$. To simplify the calculation, we assume that $\Delta_{i}$ error terms are small and they can be expanded to first order. Then the trigonometric identities can be rewritten, for example, as $C_{\phi 0} \simeq C_{\phi 0^{\prime}}-2 \Delta_{\phi 0} S_{\phi 0^{\prime}}$ and $S_{\phi 0} \simeq S_{\phi 0^{\prime}}-2 \Delta_{\phi 0} C_{\phi 0^{\prime}}$, and the measured intensity can be factored as follows:

$$
\begin{aligned}
I(t)= & I^{\prime}(t)+I_{\phi 0}(t) \Delta_{\phi 0}+I_{\phi 1}(t) \Delta_{\phi 1}+I_{\delta 0}(t) \Delta_{\delta 0} \\
& +I_{\delta 1}(t) \Delta_{\delta 1}+O\left(\Delta_{i}^{2}\right)+\cdots,
\end{aligned}
$$

where $I^{\prime}(t)$ is the intensity that would be measured if the orientation and retardation of the compensators were known with total precision $\left(\Delta_{i}=0\right) . I_{\phi 0}(t)$, $I_{\phi 1}(t), I_{\delta 0}(t)$, and $I_{\delta 1}(t)$ are the first-order functions that are linear with the calibration errors and the expansion still has many other higher-order terms.

Figure 3 shows how a measured calibration MM deviates from the ideal diagonal matrix when the error terms $\Delta_{i}$ take values in the interval $\pm 5^{\circ}$. The matrix elements $m_{03}, m_{13}, m_{23}, m_{30}, m_{31}$, and $m_{32}$ are unaffected by any $\Delta_{i}$ so their determination is always robust regardless of a bad calibration. $m_{01}$ and $m_{10}$ are, respectively, preferentially sensitive to $\Delta_{\delta 1}$ and $\Delta_{\delta 0} . \Delta_{\theta 1}$ is the only error term affecting $m_{02}$ while $\Delta_{\theta 0}$ is the only contributor to $m_{20}$. The figure shows
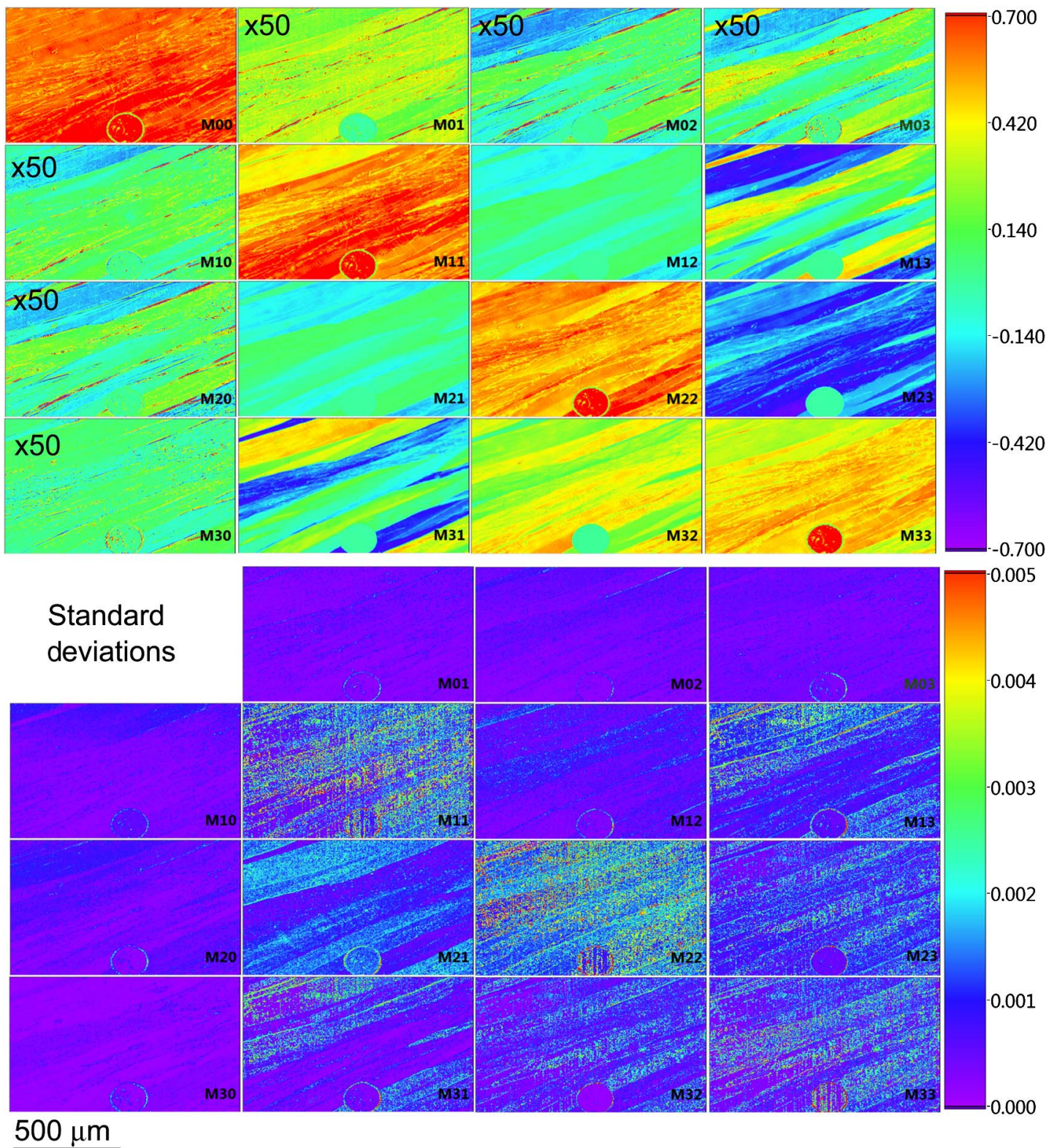

Fig. 4. Measurement of a polycrystalline benzil sample at $440 \mathrm{~nm}$. The sample is a crystallization from the melt sandwiched between glass covers. The bottom image shows the standard deviations of the normalized MM after five measurements. The numbers appearing in some MM elements indicate the multiplying factors used to enhance the values of these elements for better visibility. 
that $m_{12}$ and $m_{21}$ are very sensitive to $\Delta_{\theta 0}$ and $\Delta_{\theta 1}$, but both contributions cannot be separated in these MM elements, and therefore they are not a good election for calibration. The diagonal elements have considerable nonlinear error contributions and therefore they are neither suitable for calibration. Given these results, it becomes clear that the best MM elements to be used for calibration are $m_{01}, m_{02}, m_{10}$, and $m_{20}$ :

$$
\mathbf{M}_{\text {calib }}=\left[\begin{array}{cccc}
\bullet & \propto \Delta_{\delta_{0}} & \propto \Delta_{\theta_{0}} & \bullet \\
\propto \Delta_{\delta_{1}} & \bullet & \bullet & \bullet \\
\propto \Delta_{\theta_{1}} & \bullet & \bullet & \bullet \\
\bullet & \bullet & \bullet & \bullet
\end{array}\right] .
$$

As the dependence of these MM elements with uncertainties that are linear and uncoupled, the optimal values of $\phi_{0}, \phi_{1}, \delta_{0}$, and $\delta_{1}$ are deduced independently. From the results of Fig. 3 it follows that a poor calibration of $\phi_{0}$ and $\phi_{1}$ will immediately translate into large errors in $m_{12}$ and $m_{21}$ as these matrix elements are very sensitive to these phases.

As a further refinement of the calibration procedure, the small perturbation that the strains of the objective may introduce in the measurement can be corrected if a preliminary measurement without sample is made. This allows us to determine the MM image of the objective, $\mathbf{M}_{\text {obj }}$. As in the optical train of the instrument, the objective is right after the sample, the small perturbation effect of the objective can be corrected with the following operation:

$$
\mathbf{M}_{\text {corrected }}=\mathbf{M}_{\mathrm{obj}}^{-1} \mathbf{M}_{\text {measured }} \text {. }
$$

As the strains of the objective are not homogenous across the image, every pixel camera can have a slightly different $\mathbf{M}_{\mathrm{obj}}$.

\section{Measurement}

The measurement process is similar to any other microscope. The sample is introduced in the sample holder and its position is adjusted until it is in focus. Before starting the data collection it is convenient to adjust the exposure of the camera to a value high enough to use all the digital dynamic range offered by the camera (eight bits in our case), although at the same time it must be verified that the intensity is not saturated for any position of the compensators. After the exposure time has been adjusted to an optimum value, the data collection can start. With the parameters described in the previous sections the data collection takes $72 \mathrm{~s}$ and the data processing adds around 10 extra seconds. To improve the signalto-noise ratio of the MM image, the measurement may be repeated several times. This permits us to average the results and compute the standard deviation as an error estimator of the measurement.
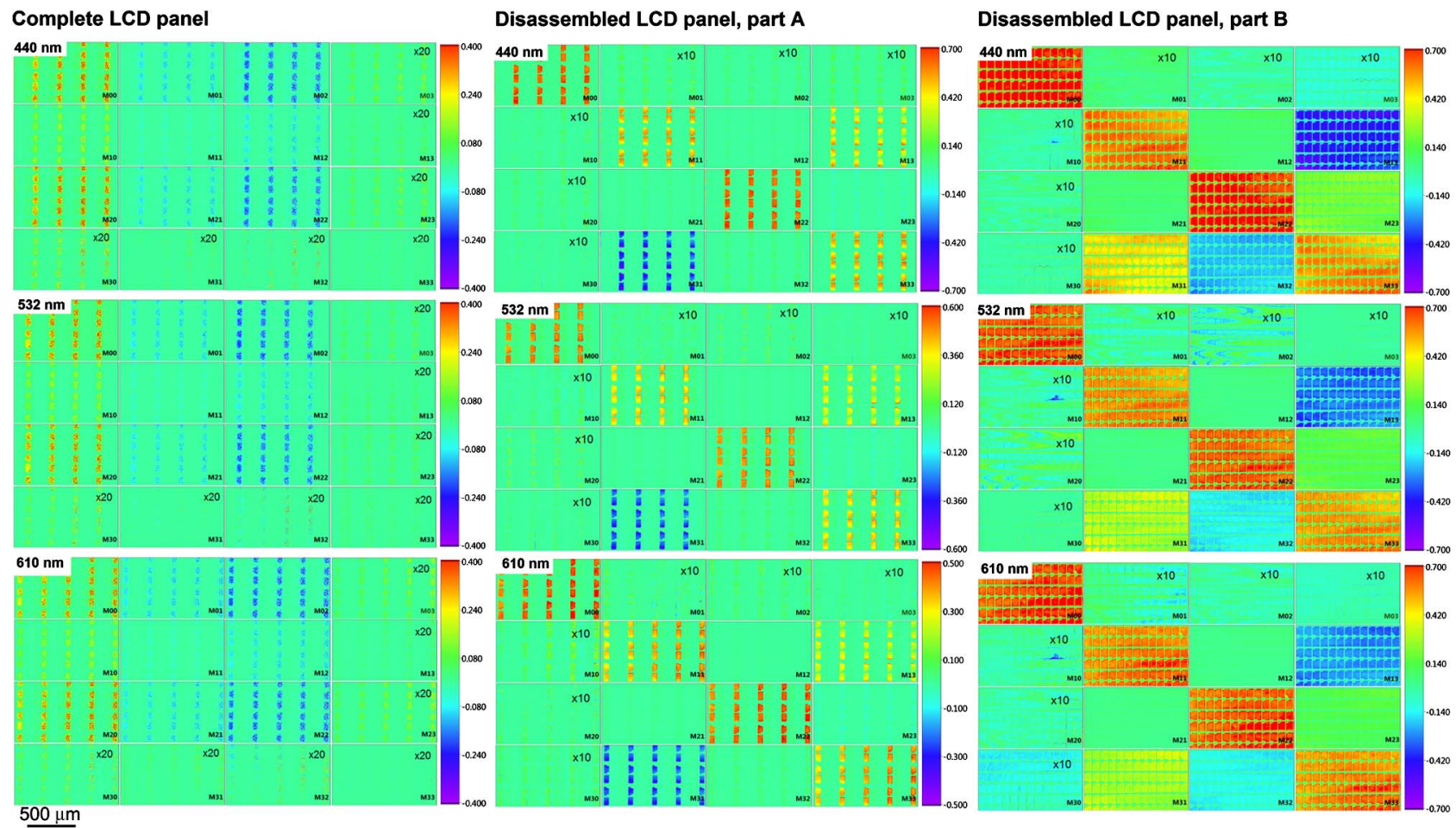

Fig. 5. Comparison of the optical response of the different parts of an LCD panel at the subpixel level. The comparison include three different wavelengths that selectively activate certain subpixels. In the first column light transmitted through the complete LCD panel is studied. The Mueller matrices in the second and third columns correspond to measurements made after disassembling the two glass panels that sandwich the liquid crystal and removing the two film polarizers. Part A includes the color filter that defines the RGB subpixel pattern and also contains rectangular electrodes for every subpixel. Part B shows the semi-transparent transistors arranged in a square lattice. 


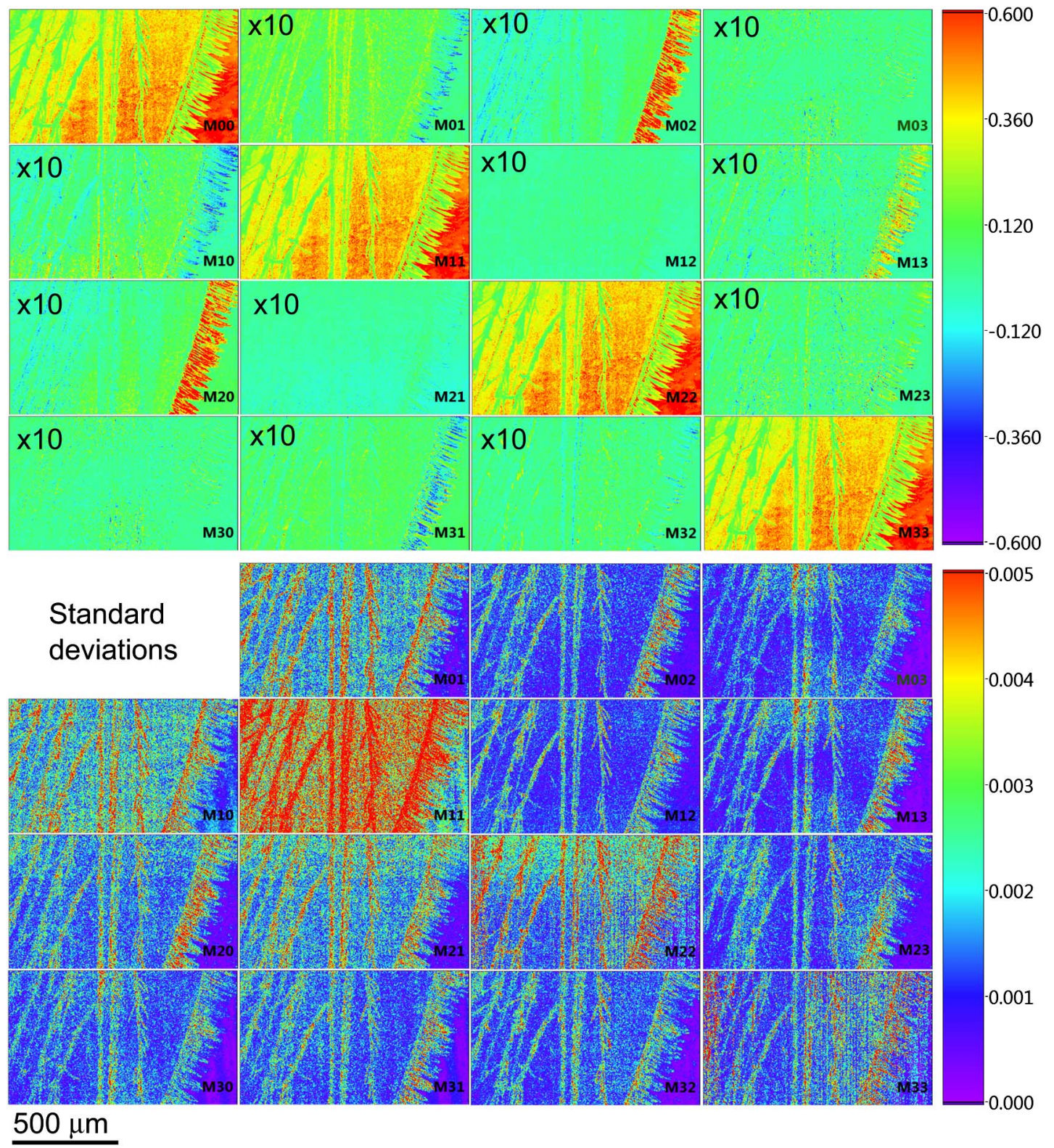

Fig. 6. Detail of an MM measurement of a mosquito wing at $610 \mathrm{~nm}$. The numbers appearing in some MM elements indicate the multiplying factors used to enhance the values of these elements for better visibility.

Mueller matrices are usually presented with their elements normalized to the element $m_{00}$. All the information about the anisotropy and/or depolarization of a specimen is contained in its normalized MM. However, some structural features of the samples that are neither anisotropic nor depolarizing can be obscured by the normalization, and therefore it is convenient to keep a MM of microscopy unnormalized.

Figure 4 shows the MM measurement of a thin polycrystalline layer of benzil crystallized from the melt along with the standard deviations of the corresponding normalized MM after five measurements. Benzil is a common organic compound with yellowish appearance and with an absorption band centered around $390 \mathrm{~nm}$ [28]. This measurement was done at $440 \mathrm{~nm}$ and, although at this wavelength the crystals are almost fully transparent, the small but not fully negligible values of the MM elements $m_{01}, m_{02}, m_{03}, m_{10}, m_{20}$, and $m_{30}$ are due to some traces of the linear and circular dichroism that is still detectable at the edge of the absorption band. The plotted standard deviations are better than 0.005 for all the pixels of any MM element.

An LCD display is rich in optical components that alter the polarization of light and can be easily investigated with the MM microscope. We have studied a computer LCD panel (AU Optronics M170EC01) at various steps of disassembly (Fig. 5). Light of three different wavelengths $(440,532$ and $610 \mathrm{~nm})$ has been used to activate the three RGB color subpixels present in the panel. When the LCD is complete 
(first column in the image) the measured MM is governed by the two crossed polarizing films glued to both sides of the panel. In this case light transmission is possible because the nematic liquid crystal of the display modifies the polarization of light after it goes through the first polarizer. The MMs in the second and third columns of Fig. 5 correspond to the measurements of each one of the glass plates, $\mathrm{A}$ and $\mathrm{B}$, that sandwich the liquid crystal. Here the film polarizers had been already removed and the surface of both plates was still soaked with the nematic liquid crystal. In plate A there is the RGB color filter that assigns a color to every subpixel while the rectangular structures are electrodes. The optical response of plate B is quite achromatic because it does not have any color filter and the structures, organized following a square lattice, are the semi-transparent transistors. The linear birefringence is due to the liquid crystal. Interestingly, the signs of the elements of the MM clearly indicate that the relative orientation of the liquid crystal in parts A and B is approximatively $90^{\circ}$. This is not too surprising because each one of the glass plates contains a alignment layer with grooves that serve to align the liquid crystal that is in contact with that surface. The misorientation of the grooves of part A and part $\mathrm{B}$ is $90^{\circ}$ to force the liquid crystal molecules to adopt a helical configuration when they are compressed between the two surfaces. In this configuration the unpowered liquid crystal produces an optical

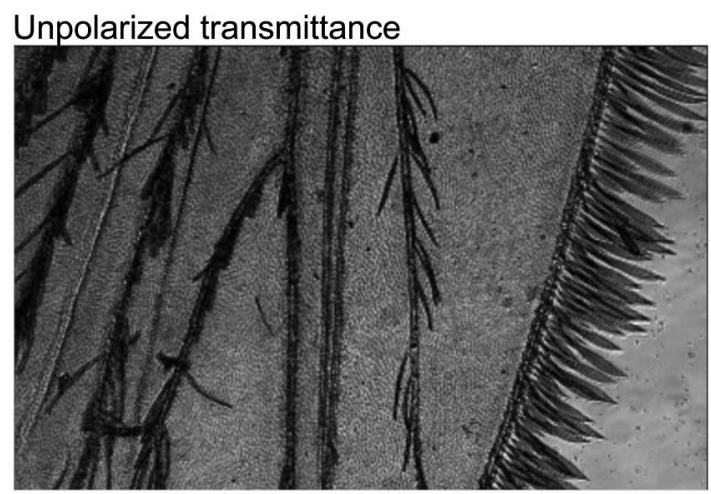

Linear dichroism (rad)

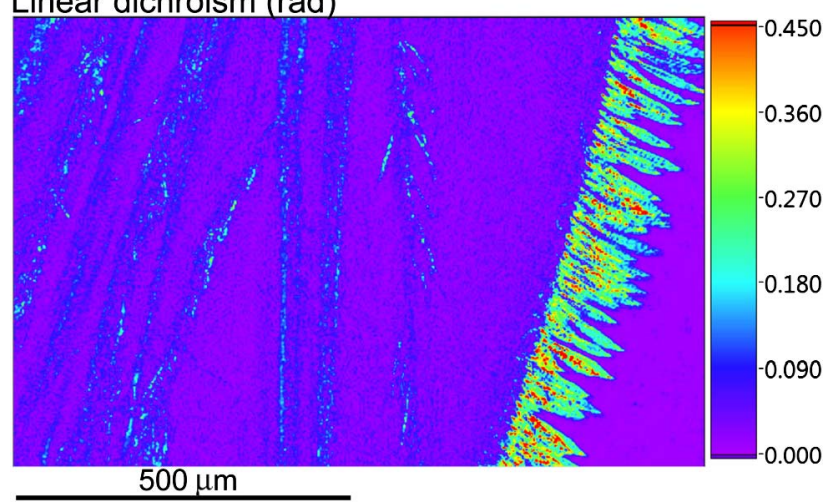

Fig. 7. Unpolarized transmittance (MM element $m_{00}$ ) and linear dichroism image of a part of the mosquito wing. A great contrast is obtained in the fringes located in the outer part of the wing. rotation of around $90^{\circ}$, matching the misorientation of the two film polarizers and, therefore, allowing maximum light transmittance.

As an example of another application of the MM microscope, we show in Fig. 6 the MM and the standard deviation corresponding to a mosquito wing examined at $610 \mathrm{~nm}$ with a $\times 10$ objective. The MM shows some interesting features, for example, some structures show relatively high linear birefringence and linear dichroism. It is specially visible in the fringe scales that are in the wing margin (right part of the images). This is most likely due to the presence of some oriented dye molecule in the scales. Figure 7 shows the linear dichroism in radians as calculated from the MM following the method indicated in [7]. The scales in the wing margin are highly contrasted due to their high linear dichroism and some slight linear dichroism is also appreciable in the wing veins.

\section{Summary and Conclusions}

We have presented a novel MM microscope built in our laboratory. The instrument uses two continuously rotating compensators to simultaneously obtain the 16 elements of an MM. Some features that make this instrument distinct are:

1. An absolute accuracy better than $0.5 \%$ is achieved for all elements of the MM in the whole image.

2. A typical measurement takes around one minute.

3. The calibration is self-consistent and does not require any reference sample.

4. With a $50 \times$ objective it can resolve objects of $1-2 \mu \mathrm{m}$ in size

5. It is suitable for spectroscopic measurements because it does not require specific values of the retardation of the compensators.

6 . It is relatively inexpensive to construct and operate because it does not require ultra-fast cameras or high-performance rotation stages.

In conclusion, we have demonstrated that the MM microscope is a very versatile instrument that can be used for a wide range of popular applications in which imaging the optical anisotropy of a sample is essential. As all the elements of the MM are measured, a single measurement is usually sufficient to obtain a complete and quantitative determination of all the optical properties of a sample, even for anisotropic specimens with a high degree of complexity. It also characterizes depolarization phenomena. Compared to other MM imaging devices, the MM microscope offers a higher sensitivity as its measurement principle is based in digital demodulation.

O. A. acknowledges financial support from a Beatriu de Pinós Fellowship (2011 BP-B 00059). Part of the experiment was financially supported by the AGAUR of the Generalitat de Catalunya, project 2009SGR00185. 


\section{References}

1. D. B. Murphy, Fundamentals of Light Microscopy and Electronic Imaging, 1st ed. (Wiley-Liss, 2001).

2. R. L. Long, M. P. Bange, S. G. Gordon, and G. A. Constable, "Measuring the maturity of developing cotton fibers using an automated polarized light microscopy technique," Text. Res. J. 80, 463-471 (2010).

3. D. B. Murphy, Handbook of Microscopy: Applications in Materials Science, Solid-State Physics and Chemistry, vol. 3 (Wiley-VCH, 1996).

4. R. Weaver, "Rediscovering polarized light microscopy," Am Lab 35, 55-61 (2003).

5. S. Ross, R. Newton, Y. M. Zhou, J. Haffegee, M. W. Ho, J. P. Bolton, and D. Knight, "Quantitative image analysis of birefringent biological material," J. Microsc. 187, 62-67 (1997).

6. O. Arteaga, "Mueller matrix polarimetry of anisotropic chiral media," Ph.D. thesis, University of Barcelona (2010).

7. O. Arteaga and A. Canillas, "Analytic inversion of the MuellerJones polarization matrices for homogeneous media," Opt. Lett. 35, 559-561 (2010).

8. J. Schellman and H. P. Jensen, "Optical spectroscopy of oriented molecules," Chem. Rev. 87, 1359-1399 (1987).

9. R. Ossikovski, "Differential matrix formalism for depolarizing anisotropic media," Opt. Lett. 36, 2330-2332 (2011).

10. O. Arteaga and B. Kahr, "Characterization of homogenous depolarizing media based on Mueller matrix differential decomposition," Opt. Lett. 38, 1134-1136 (2013).

11. O. Arteaga, "Number of independent parameters in the Mueller matrix representation of homogeneous depolarizing media," Opt. Lett. 38, 1131-1133 (2013).

12. E. Bernabeu and J. J. Gil, "An experimental device for the dynamic determination of Mueller matrices," J. Opt. 16, 139-141 (1985).

13. K. Ichimoto, K. Shinoda, T. Yamamoto, and J. Kiyohara, "Photopolarimetric measurement system of Mueller matrix with dual rotating waveplates," Publ. Nat. Ast. Obs. J. 9, 11-19 (2006).

14. O. Arteaga, J. Freudenthal, B. Wang, and B. Kahr, "Mueller matrix polarimetry with four photoelastic modulators: theory and calibration,” Appl. Opt. 51, 6805-6817 (2012).
15. A. De Martino, Y.-K. Kim, E. Garcia-Caurel, B. Laude, and B. Drévillon, "Optimized Mueller polarimeter with liquid crystals," Opt. Lett. 28, 616-618 (2003).

16. R. M. A. Azzam, "Division-of-amplitude photopolarimeter (DOAP) for the simultaneous measurement of all four stokes parameters of light," Opt. Acta 29, 685-689 (1982).

17. D. Lara and C. Dainty, "Axially resolved complete Mueller matrix confocal microscopy," Appl. Opt. 45, 1917-1930 (2006).

18. S. Alali and I. A. Vitkin, "Optimization of rapid Mueller matrix imaging of turbid media using four photoelastic modulators without mechanically moving parts," Opt. Eng. 52, 103114 (2013).

19. J. Freudenthal and B. Wang, Hinds Instruments, Hillsboro, Oregon 97124 (personal communication, 2013).

20. P. S. Hauge, "Mueller matrix ellipsometry with imperfect compensators," J. Opt. Soc. Am. 68, 1519-1528 (1978).

21. J. J. Gil, "Método dinámico de determinación de parámetros de Stokes y matrices de Mueller por análisis de Fourier," Master's thesis (Universidad de Zaragoza, 1979).

22. R. M. A. Azzam, "Photopolarimetric measurement of the Mueller matrix by Fourier analysis of a single detected signal," Opt. Lett. 2, 148-150 (1978).

23. J. H. Freudenthal, E. Hollis, and B. Kahr, "Imaging chiroptical artifacts," Chirality 21, S20-S27 (2009).

24. S. Ben Hatit, M. Foldyna, A. De Martino, and B. Drévillon, "Angle-resolved Mueller polarimeter using a microscope objective," Phys. Stat. Sol. (a) 205, 743-747 (2008).

25. L. M. S. Ars, P. G. Ellingsen, and M. Kildemo, "Near infra-red Mueller matrix imaging system and application to retardance imaging of strain," Thin Solid Films 519, 2737-2741 (2011).

26. D. H. Goldstein, "Mueller matrix dual-rotating retarder polarimeter," Appl. Opt. 31, 6676-6683 (1992).

27. R. W. Collins and J. Koh, "Dual rotating-compensator multichannel ellipsometer: instrument design for real-time Mueller matrix spectroscopy of surfaces and films," J. Opt. Soc. Am. A 16, 1997-2006 (1999).

28. Z. El-Hachemi, O. Arteaga, A. Canillas, J. Crusats, A. Sorrenti, S. Veintemillas-Verdaguer, and J. M. Ribo, "Achiral-to-chiral transition in benzil solidification: analogies with racemic conglomerates systems showing deracemization," Chirality 25, 393-399 (2013). 